\title{
Design and Implementation of DLNA DMS Through IEEE1394*
}

\author{
Gu Su Kim ${ }^{1}$, Chul-Seung Kim ${ }^{1}$, Hyun-Su Jang ${ }^{1}$, Moon Seok Chang ${ }^{2}$, \\ and Young Ik Eom ${ }^{1}$ \\ ${ }^{1}$ School of Information and Communication Engineering, \\ Sungkyunkwan University, 300 Chunchun-dong, Jangan-gu, Suwon, \\ Kyunggi-do, 440-746, Korea \\ \{gusukim, hallower, jhs4071, yieom\} @ece.skku.ac.kr \\ 2 Advisory S/W Engineer Kernel Performance Server Group, \\ MS 957111400 Burnet Rd. Austin, TX 78758, USA \\ moon@austin.ibm.com
}

\begin{abstract}
With the technological growth of the AV(Audio and Video) equipments and the popularization of video contents, IEEE1394 for the transmission of AV data has spread fast. However, DLNA(Digital Living Network Alliance), which is a standard of the home network middleware, does not consider the transmission of AV data over IEEE1394. In this paper, we propose a scheme for transmission of AV contents over IEEE1394 in DLNA environments. In addition, by the validation test of DLNA compatibility, we show that our proposed scheme can support AV contents sharing without regard to the manufactures and transmission media.
\end{abstract}

Keywords: AV, DLNA, IEEE1394.

\section{Introduction}

With the progress of various IT technologies and the proliferation of high speed access networks and digital convergence, the concern on home network environments has increased and many home network middleware technologies and standards have been presented [1. Digital Living Network Alliance(DLNA) presents the Home Network Device Interoperability Guidelines v1.0 for sharing the AV (Audio and Video) contents 23. However, these guidelines support only HTTPGET method as the basic protocol for transmission of AV contents and do not consider IEEE1394 that is widely being used in devices of home network environments 415. Therefore, the AV devices using IEEE1394 transmit AV contents through manufacturer specific methods in DLNA environments and there are problems when different manufacturer devices share the AV contents.

In this paper, we propose IEEE1394 DMS(Digital Media Server) architecture for DLNA environments and show the implementation of the proposed IEEE1394

\footnotetext{
* This research was supported by MIC, Korea under ITRC IITA-2006-(C10900603-0046).
} 
DMS architecture and test whether our IEEE1394 DMS prototype has the compatibility with DLNA standard.

The rest of the paper is organized as follows. In Section 2, we briefly show the design of our proposed IEEE1394 DMS architecture. Section 3 presents the experimental results focusing on the DLNA compatibility. Finally, Section 4 concludes with a summary.

\section{The Architecture of IEEE1394 DMS}

The proposed IEEE1394 DMS architecture uses the IEEE1394 as the basic transmission interface and consists of Content Creation Subsystem, Content Management Subsystem, Content Storage Database, and Content Transfer Subsystem. Figure 1 shows the IEEE1394 DMS architecture.

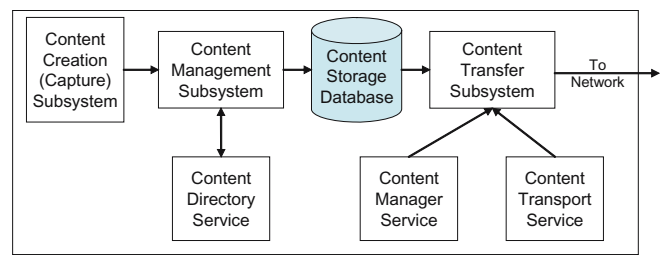

Fig. 1. IEEE1394 DMS architecture

We constructed the software stack of our IEEE1394 DMS architecture which consists of IEEE1394 interface, IEEE1394 device driver, IEEE1394 library, IPv4 over IEEE1394 driver, TCP/IP stack, UPnP DA(Device Architecture)/AV stack, and DMS applications.

DLNA device using the IEEE1394 interface can get the information for the transmission of AV contents through the device description. The device description expresses that the device has the GUID number of IEEE1394 interface and plays the role of DLNA DMS and provides the service of UPnP DA stack and Contents Directory, Connection Manager, and AV Transport Service of UPnP AV stack 677. In addition to these basic information, Our URI for IEEE1394 DMS using IEC61883 protocol provides additional information as follows: the bandwidth of IEEE1394, the GUID of Isochronous Resource Manager(IRM) assigning the isochronous channel, the contents type, the GUID of IEEE1394 DMS, and PCR number.

\section{Implementation and Experiment Results}

We implemented IEEE1394 DMS on Redhat 9 Linux platform and used IEEE1394 driver for Linux[8] and Eth1394 module, which is IPv4 over IEEE1394 driver 9]. Also, we used UPnP stack of twonkyvision [10, whose DMS is compatible with DLNA. 


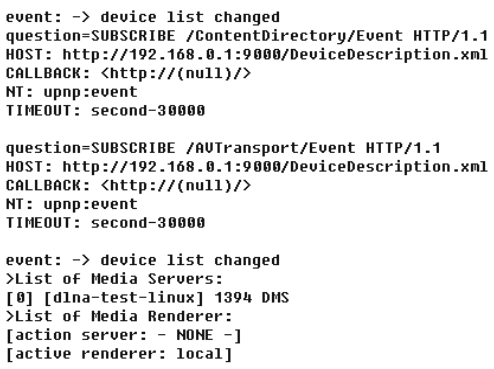

Fig. 2. URI for IEC61883 protocol

Figure 2 shows the result that IEEE1394 DMP confirms IEEE1394 DMS and its service, and IEEE1394 DMS was registered as a media server.

Figure 3 shows the process that DMP selects and plays an AV content. In Figure 3, the first paragraph shows the result that DMP performs AVT: SetAVTra nsportURI action including URI (iec61883://00601d00000006bd:63/disk/video/ O37.mpg?DLNA.ORG_PN=MPEG_PS_NTSC;DLNA.OR G_OP=01) for selection of the content. The second paragraph shows the response of the SetAVTransportURI action and the third paragraph shows AVT:Play action. The last paragraph shows the response from the AVT:Play action.

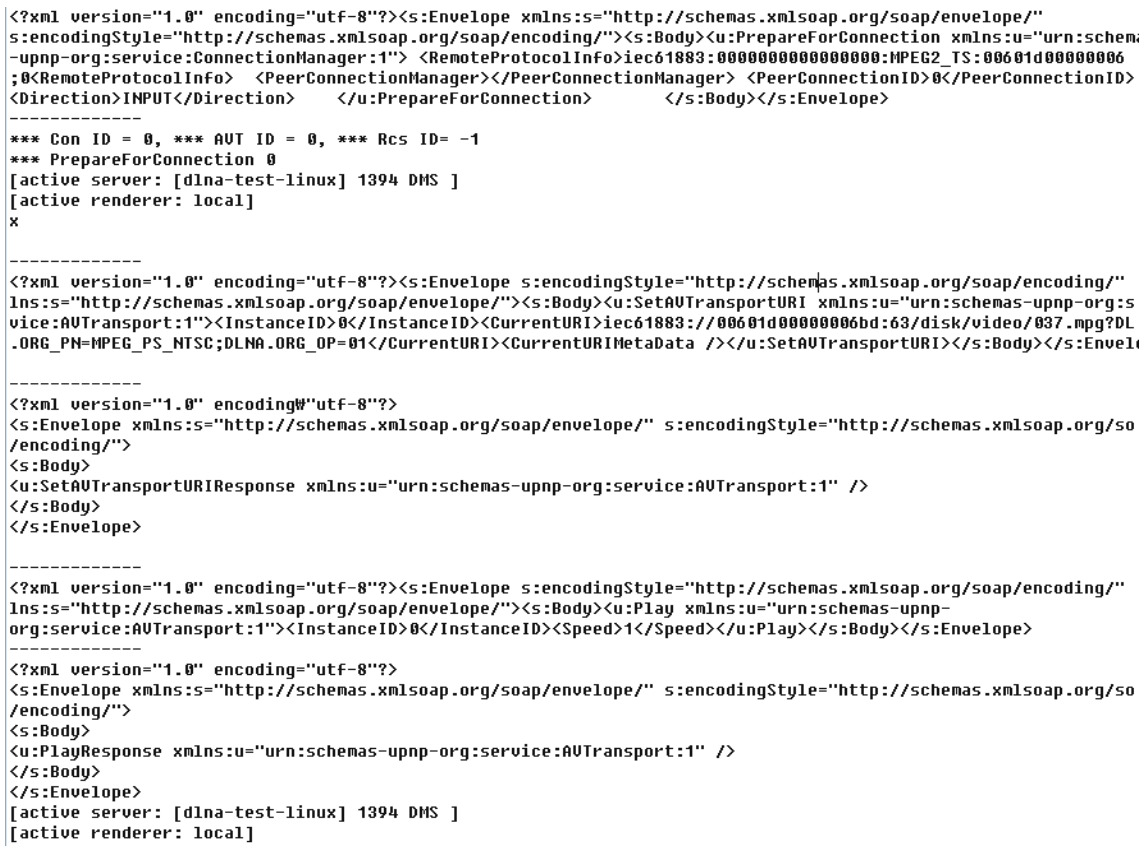

Fig. 3. URI for IEC61883 protocol 
In order to verify that our implemented IEEE1394 DMS has compatibility with DLNA, we tested our system through DLNA Conformance Test Tool(CTT) 11. Our test items are as follows: device description, URI, DMS, contents directory service, connection manager service, GetProtocolInfo of connection manager service, contents browsing, Protocollnfo, and MM URI item.

In the CTT verification, our system satisfied above items except for GetProtocolInfo and ProtocolInfo items. GetProtocolInfo and ProtocolInfo items are failed in our tests because the current DLNA specification supports only HTTPGET protocol and does not support IEC61883 protocol. In order to solve these failures in CTT test, it is needed that the future DLNA 2.0 will have to define IEEE1394 as transmission protocol and CTT will have to reflect the test items for IEEE1394.

\section{Conclusion}

In this paper, we proposed the architecture of IEEE1394 DMS for sharing of AV contents in DLNA envrionments and implemented the device description for IEEE1394 DMS, AV Transport Service, and URI for content specification over IEEE1394. Also, we showed that our proposed scheme is compatible with DLNA by testing through DLNA CTT. Our proposed IEEE1394 DMS allow devices only with IEEE1394 interface such as TV, STB, and DVD player, to share AV contents on DLNA environments.

\section{References}

1. T. Nakajima and I. Satoh, "A software infrastructure for supporting spontaneous and personalized interaction in home computing environments," Personal and Ubiquitous Computing, Springer-Verlag, Vol. 10, No. 6, pp. 379 - 391, Sep. 2006.

2. DLNA Homepage, http://www.dlna.org/.

3. DLNA, Home Networked Device Interoperability Guidelines v1.0, Jun. 2004.

4. IEEE Std 1394a-2000, IEEE Standard for a High Performance Serial Bus-Amendment 1, Mar. 2000.

5. IEEE Std 1394b-2002, IEEE Standard for a High-Performance Serial BusAmendment 2, 2002.

6. UPnP Forum, UPnP Device Architecture 1.0, May 2003.

7. UPnP Forum, UPnP AV Architecture 0.83, Jun. 2002.

8. IEEE1394 for Linux, http://www.linux1394.org/

9. IPv4 over IEEE1394 (RFC2734), http://www.ietf.org/rfc/rfc2734.txt?number $=2734$

10. http://www.twonkyvision.com/index.html

11. DLNA Conformance Test Tool, http://www.dlna.org/members/ctt/ 